\title{
Positron Fraction from Dark Matter Annihilation in the CMSSM
}

\author{
W. de Boer ${ }^{\mathrm{a}}$ C. Sander ${ }^{\mathrm{a}}$, M.Horn ${ }^{\mathrm{a}}$ and D. Kazakov ${ }^{\mathrm{b}}$ \\ anstitut für Experimentelle Kernphysik, Universität Karlsruhe (TH), \\ P.O. Box 6980, 76128 Karlsruhe, Germany \\ ${ }^{\mathrm{b}}$ Bogoliubov Laboratory of Theoretical Physics, JINR, \\ 141980 Dubna, Moscow Region, Russian Federation
}

\begin{abstract}
We investigate, if the cosmic ray positron fraction, as reported by the HEAT and AMS collaborations, is compatible with the annihilation of neutralinos in the supergravity inspired Constrained Minimal Supersymmetric Model (CMSSM), thus complementing previous investigations, which did not consider constraints from unification, electroweak symmetry breaking and the present Higgs limits at LEP. We scan over the complete SUSY parameter space and find that in the acceptable regions the neutralino annihilation into $b \bar{b}$ quark pairs is the dominant channel and improves the fit to the experimental positron fraction data considerably compared to a fit with background only. These fits are comparable to the fit for regions of parameter space, where the annihilation into $W^{+} W^{-}$pairs dominates. However, these latter regions are ruled out by the present Higgs limit of $114 \mathrm{GeV}$ from LEP.
\end{abstract}

\section{Introduction}

The cosmic ray positron fraction at momenta above $7 \mathrm{GeV}$, as reported by the HEAT collaboration, is difficult to describe by the background only hypothesis [1]. The data at lower momenta agree with previous data from the AMS experiment [2]. A contribution from the annihilation of neutralinos, which are the leading candidates to explain the cold dark matter in the universe, can improve the fits considerably [3, 1 . The neutralinos are the Lightest Supersymmetric Particles (LSP) in supersymmetric extensions of the Standard Model, which are stable, if R-parity is conserved. This new multiplicative quantum number for the supersymmetric partners of the Standard Model (SM) particles is needed to prevent proton decay and simultaneously prevents the LSP a) to decay into the lighter SM particles and b) can only interact with normal matter by producing additional supersymmetric particles. The cross sections for the latter are typically of the order of the weak cross sections, so the LSP is "neutrinolike", i.e. it would form halos around the galaxies and consequently, it is an excellent candidate for dark matter.
In addition to being of interest for cosmology, supersymmetry solves also many outstanding problems in particle physics, among them[5]: 1) it provides a unification of the strong and electroweak forces, thus being a prototype theory for a Grand Unified Theory (GUT) 2) it predicts spontaneous electroweak symmetry breaking (EWSB) by radiative corrections through the heavy top quark, thus providing a relation between the GUT scale, the electroweak scale and the top mass, which is perfectly fullfilled 3) it includes gravity 4) it cancels the quadratic divergencies in the Higgs mass in the SM 5) the lightest Higgs mass can be calculated to be below 125 $\mathrm{GeV}$ in perfect agreement with electroweak precision data, which prefer indeed a light Higgs mass. Therefore it is interesting to study the positron fraction from neutralino annihilation in the reduced region of SUSY parameter space, where these constraints are satisfied and compare it with the AMS and HEAT data, as will be done in the next section. 


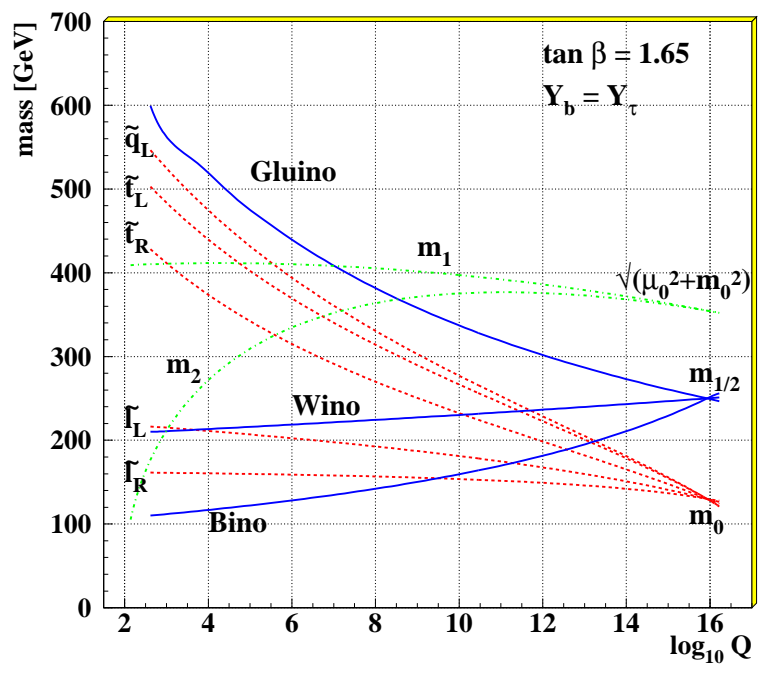

Figure 1. The running of the masses between the GUT scale and the electroweak scale.

\section{Neutralino Annihilation in the CMSSM}

In the Constrained Minimal Supersymmetric Model (CMSSM) with supergravity mediated breaking terms all sparticle masses are related by the usual GUT scale boundary conditions of a common mass $m_{0}$ for the squarks and sleptons and a common mass $m_{1 / 2}$ for the gauginos. The parameter space, where all low energy constraints are satisfied, is most easily determined by a global statistical analysis, in which the GUT scale parameters 1 are constrained to the low energy data by a $\chi^{2}$ minimization. We use the full NLO renormalization group equations [0 to calculate the low energy values of the gauge and Yukawa couplings and the one-loop RGE equations for the sparticle

\footnotetext{
${ }^{1}$ The supergravity inspired parameters are the GUT scale $M_{G U T}$, the common gauge coupling $\alpha_{G U T}$, the common mass scales $m_{0}, m_{1 / 2}$ for the spin 0 and spin $1 / 2$ sparticles, the trilinear couplings $A_{0}$ and Yukawa couplings $Y_{0}$ at the GUT scale of the third generation of fermions, the Higgs mixing parameter $\mu$ and $\tan \beta$, the ratio of vacuum expectation values of the neutral Higgs doublets.
}

masses with decoupling of the contribution to the running of the coupling constants at threshold.

Fig. 11 shows a typical running of the common masses from the GUT scale to low energies. The squarks and gluinos get a higher mass than the sleptons due to the gluonic contributions of the strong interactions. If $m_{1 / 2}$ is not strongly above $m_{0}$, the lightest mass is the supersymmetric partner of $\mathrm{U}(1)$ gauge boson, the bino, which mixes with the $W^{3}$ boson and spin $1 / 2$ higgsinos to neutralinos. The low energy value of the LSP is roughly 0.4 times its starting value at the GUT scale, i.e. $0.4 m_{1 / 2}$. From Fig. 11 it is obvious that if $m_{1 / 2}$ is much larger than $m_{0}$, the right handed stau becomes the LSP, but this cannot be a candidate for neutral dark matter. So from cosmology we require the LSP to be neutral, i.e. $m_{0}$ sufficiently large compared to $m_{1 / 2}$.

Also the evolution of the mass parameters in the Higgs potential are shown using the full 1loop contributions from all particles and sparticles. The large negative contributions to $m_{2}$ from the top Yukawa coupling drive it negative, thus inducing spontaneous electroweak symmetry breaking. The main parameter to get correct EWSB is the starting value of $m_{2}^{2}=\mu_{0}^{2}+m_{0}^{2}$ at the GUT scale. EWSB thus determines the value of the Higgs mixing parameter $\mu^{2}$ for a given value of $m_{0}$. It turns out that as long as $m_{0}$ and $m_{1 / 2}$ are of the same order of magnitude, EWSB requires the value of $\mu$ to be larger or of the same order of magnitude than $m_{1 / 2}$.

As a consequence, the Higgsinos are heavy compared to the LSP, so they hardly mix with the LSP, which is then practically a pure bino in the parameter space of interest, i.e. for LSP masses above $100 \mathrm{GeV}$, since lighter LSP masses do not yield positrons in the interesting momentum range covered by the HEAT experiment. The gaugino fraction is defined as $\left(N_{i, 1}^{2}+N_{i, 2}^{2}\right)$, where the coefficients determine the neutralino mixing $\tilde{\chi}_{i}^{0}=N_{i, 1} \tilde{B}+N_{i, 2} \tilde{W}^{3}+N_{i, 3} \tilde{H}_{1}^{0}+N_{i, 4} \tilde{H}_{2}^{0}$. As shown in Fig. 3 the gaugino fraction is close to one for LSP masses above $100 \mathrm{GeV}$, i.e. $m_{1 / 2}>$ $250 \mathrm{GeV}$. The gaugino fraction is important, since the neutralino properties are quite different for a pure gaugino or pure Higgsino.

Furthermore, the large values of $\mu$ imply that 

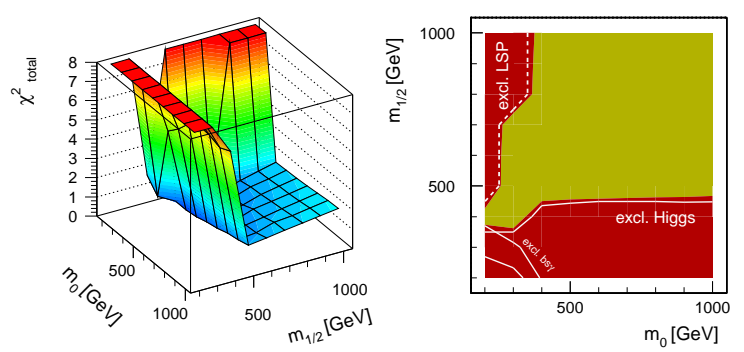

Figure 2. The allowed region (green) in the CMSSM parameter space for $\tan \beta=35$. Note that the combination of low energy constraints requires $m_{1 / 2}$ to be above $350 \mathrm{GeV}$, which corresponds to a minimum LSP mass of $150 \mathrm{GeV}$. For lower values of $\tan \beta$ the constraints are stronger, i.e. the excluded region is larger.

the pseudoscalar Higgs boson is heavy compared with the lightest Higgs boson, in which case the latter has the properties of the SM Higgs boson. The limit on the SM Higgs boson of $114 \mathrm{GeV}$ from LEP restricts the parameter $\tan \beta$ to be above $4.3[6$.

The GUT scale and Yukawa couplings are determined from the requirement of gauge unification and the masses of the third generation particles. $b-\tau$ Yukawa coupling unification can be imposed, since these fermions are in the same multiplet in any larger gauge group containing the SM gauge groups as subgroups. After running the Yukawa couplings down to low energy, one can indeed obtain the correct b-quark - and $\tau$-mass for a common Yukawa coupling at the GUT scale. But these Yukawas influence the top quark as well and the correct top quark is only obtained for $\tan \beta=1.6$ or $\tan \beta$ between 30 and 55 , where the values above 50 correspond to a positive sign of the Higgs mixing parameter $\mu$. The positive sign is preferred by the positive deviation of the anomalous magnetic moment of the muon from the SM, assuming that this $1.6 \sigma$ deviation

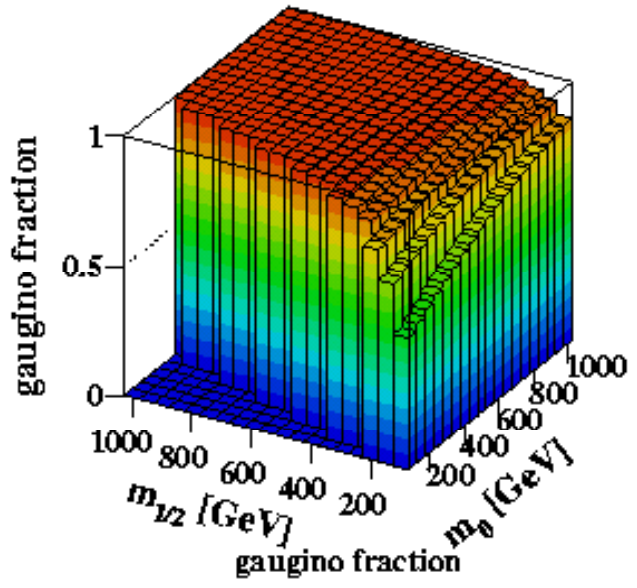

Figure 3. The gaugino fractions as function of $m_{0}$ and $m_{1 / 2}$ for $\tan \beta=35$. For lower values of $\tan \beta$ the gaugino fraction is even closer to one.

will be confirmed by future measurements. The low $\tan \beta$ solution is ruled out, since the Higgs limit of $114 \mathrm{GeV}$ at the $95 \%$ C.L. from LEP requires $\tan \beta$ to be above $4.3[$ [6], as mentioned above, so Yukawa unification suggests the high $\tan \beta$ solution.

Nevertheless, we scan over $\tan \beta$ between 1 and 50 to study the different annihilation channels, which strongly depend on $\tan \beta$. In addition we scan over the remaining parameters $m_{0}, m_{1 / 2}$. The trilinear couplings shift the Higgs mass: $A_{0}>0$ lowers the Higgs mass for given values of $m_{0}$ and $m_{1 / 2}$, so the largest excluded region is obtained for $A_{0}>0$. Positive values of $A_{0}$ are preferred by the $b \rightarrow X_{s} \gamma$ data, so if one combines all constraints (gauge unification, EWSB, $b \rightarrow X_{s} \gamma$ and $a_{\mu}$ in a single $\chi^{2}$ function, one can obtain a $95 \%$ C.L. contour for the excluded region, as shown in Fig. 2 for $\tan \beta=35$. For details we refer to previous publications [7].

Neutralino annihilation can occur through Z- and Higgs exchanges in the s-channel and sfermion exchange in the t-channel. This annihilation in the halo of the galaxies will produce 


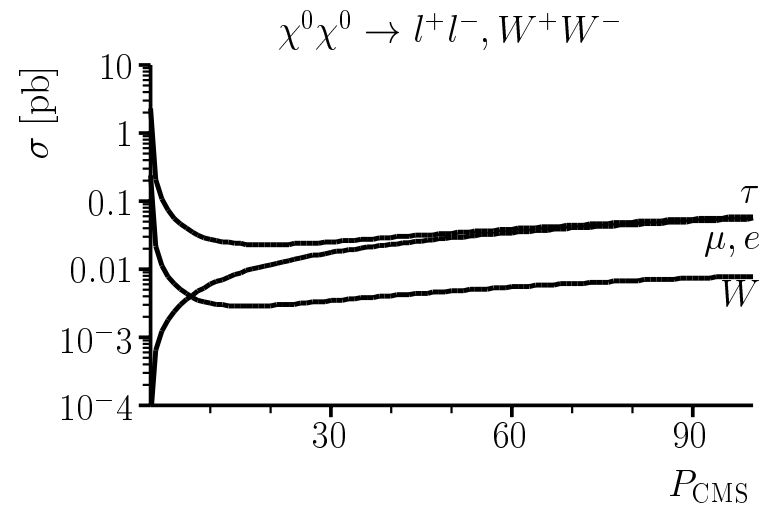

Figure 4. The neutralino annihilation total cross section as function of the center of mass momenta of the neutralinos for lepton and $W^{+} W^{-}$final states. Note the p-wave suppression at low momenta for light fermions.

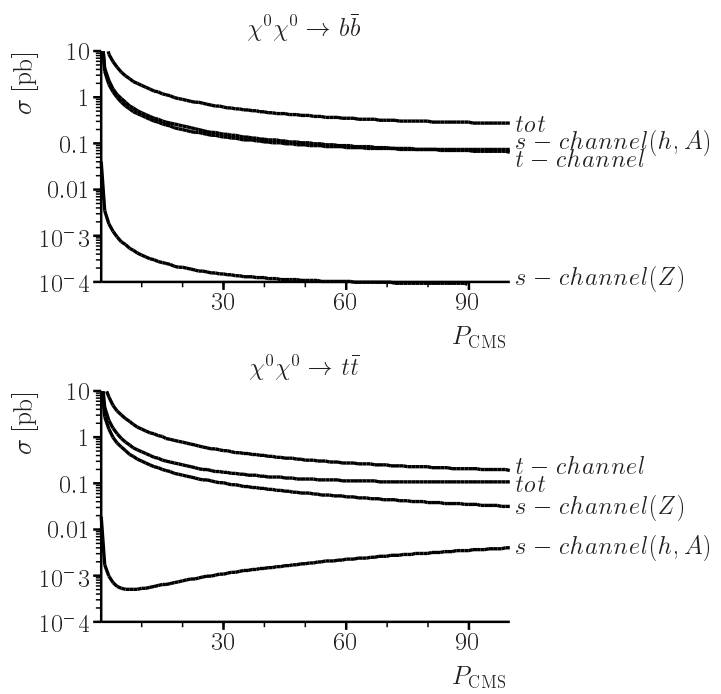

Figure 5. The neutralino annihilation cross section for various diagrams as function of the center of mass momenta of the neutralinos for $\tan \beta=35$. The curve labeled tot is the total cross section including interferences, which is larger for bottom than for top final states at large $\tan \beta$ due to the different signs of the interference terms. antimatter at high momenta, thus anomalies in the spectra of positrons and antiprotons provide an excellent signal for dark matter annihilation. The neutralino is a spin 1/2 Majorano particle, so it obeys the Pauli principle, which implies an asymmetric wave function or spins antiparallel for annihilation at rest. This results in a p-wave amplitude to the fermion-antifermion final states, which is proportional to the mass of the fermion in the final state. Therefore heavy final states are enhanced at low momenta, as demonstrated in Fig. 1 for leptons and W-bosons. The same is true for quark final states.

The total cross section is determined by the sum of the individual amplitudes; their relative signs determine the interferences. The t-channel has for t-quark and b-quark final states similar amplitudes, but the Z-exchange is for t-quarks much stronger than for b-quarks (amplitude $\propto$ mass). For Higgs exchange the b-quark final state becomes large at large $\tan \beta$ because of the coupling proportional to $\sin \beta$, while the coupling to t-quark final states, proportional to $\cos \beta$, is strongly suppressed at large $\tan \beta$. Since the Zexchange and Higgs exchange have an opposite sign, the t-quark final state is plagued by negative interferences, while the b-quark final state is enhanced by positive interferences and dominates at large $\tan \beta$. The various contributions are shown in Fig. 5 .

The total annihilation cross section is a strong function of the SUSY mass scale, since the tchannel contribution is proportional to one over the sfermion mass squared, as demonstrated in the $m_{0}, m_{1 / 2}$ plane in Fig. 6. The LSP mass is approximately $0.4 m_{1 / 2}$ and for $\tan \beta=1.6$ one observes the threshold peaks for $b \bar{b}-, W^{+} W^{-}$, and $t \bar{t}$ final states along the $m_{1 / 2}$ axis. For values of $\tan \beta>5$ the $b \bar{b}$ final state dominates and the annihilation cross sections quickly increases with $\tan \beta$, as demonstrated by the different vertical scales in the figure: at large $\tan \beta$ the cross sections are several orders of magnitude larger and keeps growing with approximately $\tan ^{2} \beta$. The boost factors needed for the best fit to describe the HEAT data at large momenta are correspondingly lower, as shown in Fig. 7. These boost fac- 

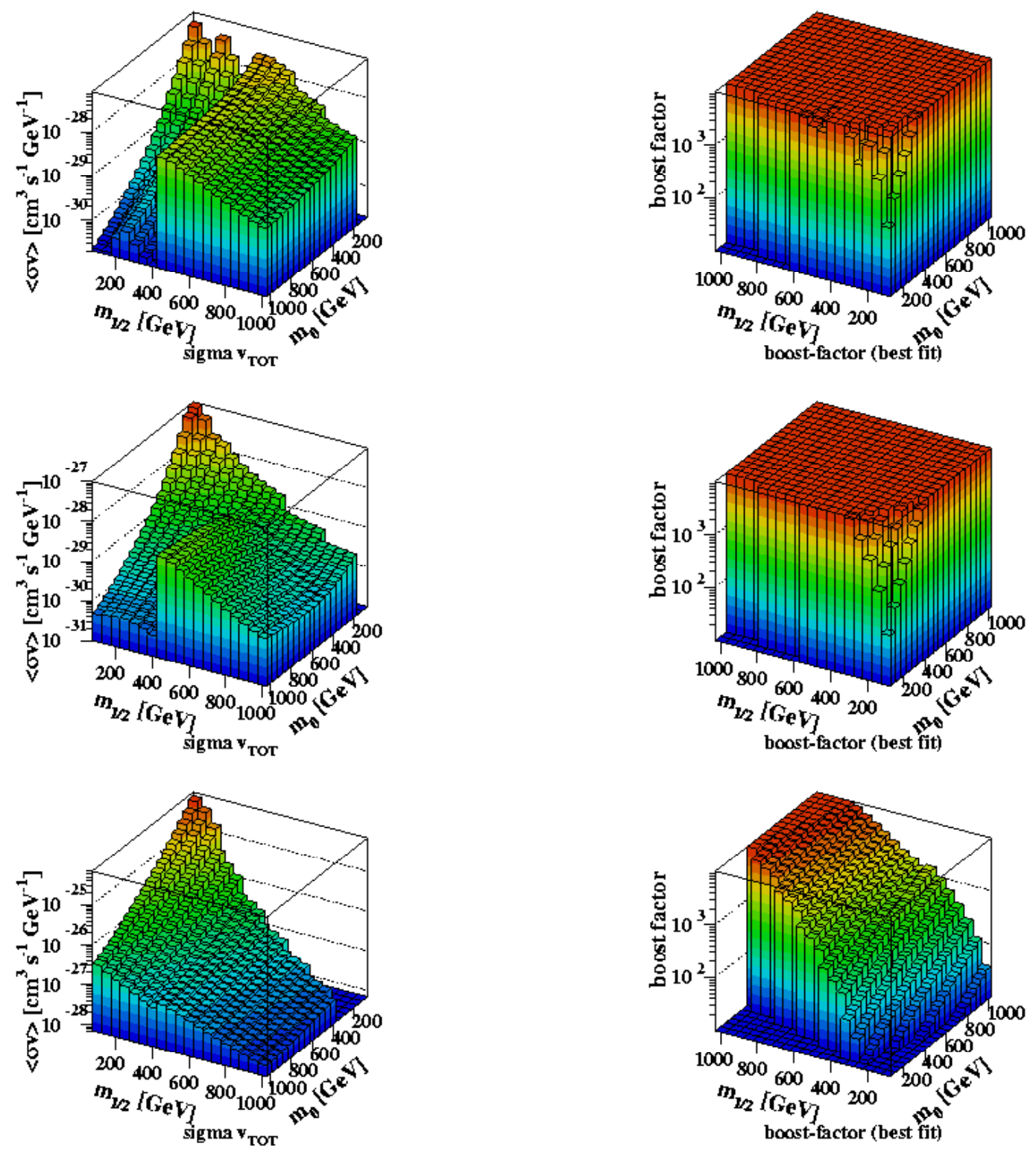

Figure $6 . \quad$ The thermally averaged cross section times velocity for neutralino annihilation as function of $m_{0}$ and $m_{1 / 2}$ for three different values of $\tan \beta$ (1.6, 5 and 35 from top.)

Figure 7. The boost factors from the best fit to the combined AMS and HEAT data as function of $m_{0}$ and $m_{1 / 2}$ for three different values of $\tan \beta$ (1.6, 5 and 35 from top). 

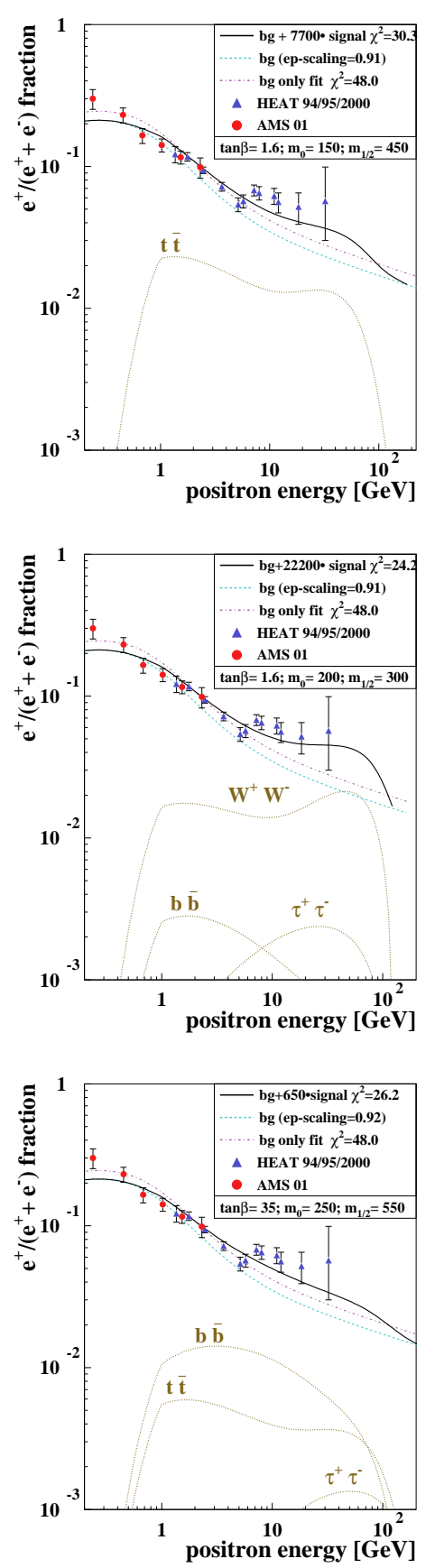

Figure 8. Fits to the data for $\tan \beta=1.6$ and LSP masses of 180 and 130 GeV (2 plots at the top) have $t \bar{t}$ and $W^{+} W^{-}$as dominant annihilation channels, but the fit for large $\tan \beta$ with $b \bar{b}$ as dominant channel yields a similar $\chi^{2}$.

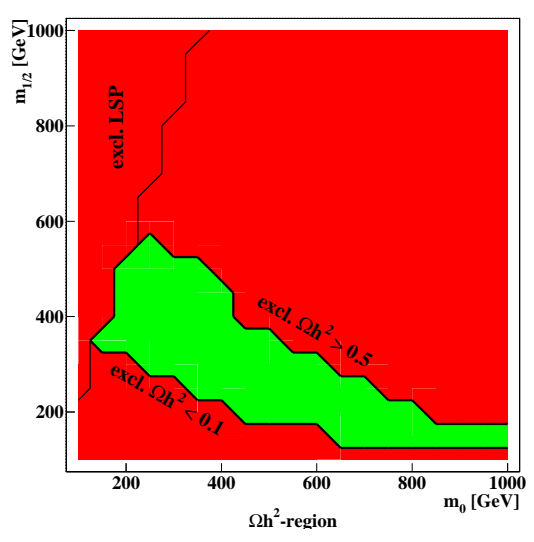

Figure 9. The preferred region (light, green) of relic density between 0.1 and 0.5 for $\tan \beta=35$, as calculated with DarkSUSY. The excluded region, where the stau would be the LSP, is also indicated. For lower value of $\tan \beta$ the green region rapidly shrinks. Coannihilation of neutralinos and staus are not considered in DarkSUSY, which would extend the green region in a narrow strip along the "excl. LSP" region.

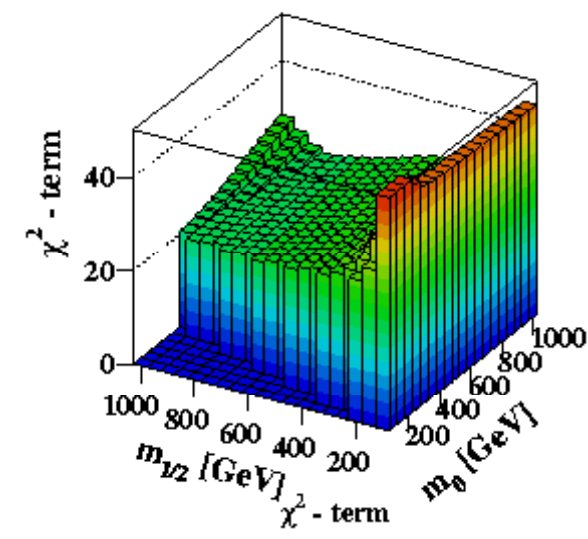

Figure 10. The $\chi^{2}$ distribution of the fit to published $A M S$ 国] and HEAT[1] data for $\tan \beta=35$. 


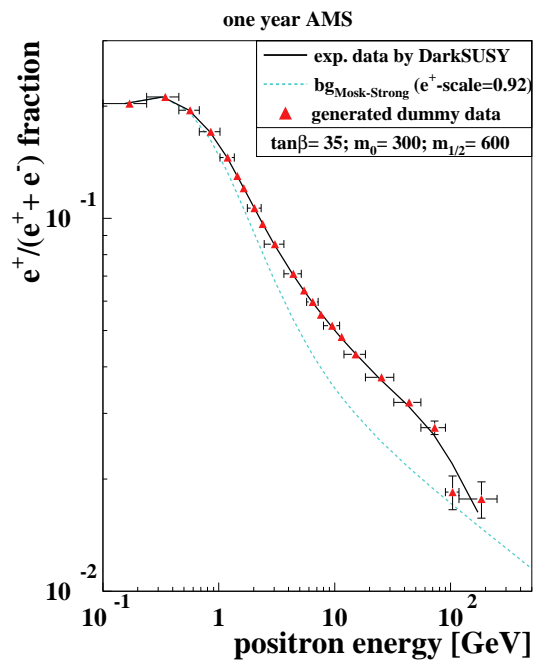

Figure 11. Expected statistics after one year of data taking with AMS-02 for a neutralino of 240 GeV and assuming a boost factor given by the best fit to the present data.

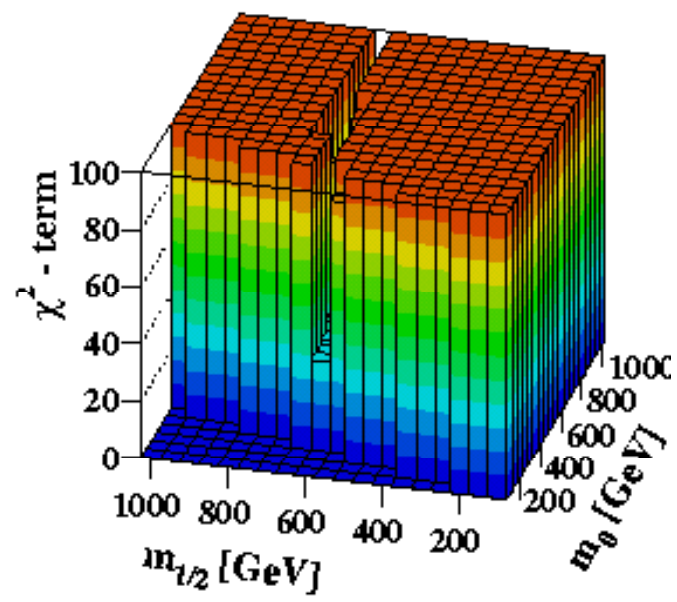

Figure 12. The $\chi^{2}$ distribution of the fit to the statistics of the future AMS-2 data of Fig. 11 tors were used in the fit as an arbitrary normalization, since the dark matter is not expected to be homogeneous, but shows some clumpiness due to gravitational interactions. Since the annihilation rate is proportional to the square of the dark matter density, the clumpiness can enhance the annihilation rate by several orders of magnitude.

The cross section calculations were done with CalcHEP [8] and DarkSUSY [9], which were found to agree within a factor of two for the $t \bar{t}$ final state and considerably better for other final states.

Fig. 8 shows the fit to the data for different regions of parameter space with different main annihilation channels. The fits were done with DarkSusy and follow the same principle as the ones from Ref. [3], i.e. the background is left free within a normalization error of about $20 \%$ and the signal can be enhanced by a boost factor as discussed above.

However, we impose the CMSSM constraints, which imply $\tan \beta>4.3$, in which case the $b \bar{b}$ final state dominates. The positron spectrum from b-quark decays can fit the present data as well as the harder decays from W-pairs, if the neutralinos are somewhat heavier, as shown by Fig. 8: the fit with b-quark final states yields a $\chi^{2} /$ d.o.f of $26 / 16$, which is not much worse than the one for $W^{+} W^{-}$final states $(24 / 16)$. These values are considerably better than the background only fit, which yields $48 / 16$. Here we used the background from Moskalenko and Strong[10], as implemented in DarkSUSY. Since the cross sections for $b \bar{b}$ final states are at least an order of magnitude above the cross sections for $t \bar{t}$ and $W^{+} W^{-}$at large $\tan \beta$, the needed boost factor for the best fit is correspondingly lower, especially for lower values of $m_{0}$, as shown before in Fig. 77. The region with large cross section and correspondingly lower boost is also the region with a relic density parameter between 0.1 and 0.5 of the critical density, as shown in Fig. 9. A range between 0.1 and 0.3 is preferred by the determination of the cosmological parameters from the red shift of distant supernovae and the acoustic peak in the microwave background 11.

The fits were repeated for all values of $m_{0}$ and $m_{1 / 2}$. The resulting $\chi^{2}$ values are plotted in Fig. 10. One observes a fast decrease in $\chi^{2}$ for val- 
ues of $m_{1 / 2}$ above $230 \mathrm{GeV}$, i.e. for LSP masses above $\approx 100 \mathrm{GeV}$. Unfortunately the data are not precise enough to prefer a certain value of the LSP mass. However,the HEAT ballon experiments correspond to only a few days of data taking. With future experiments, like PAMELA 12] on a russian satellite or AMS-02 13] on the ISS (International Space Station), one will take data for several years, thus being able to decide if the excess in the HEAT data above the present best background estimate is due to a bad knowledge of the background or if it is really a signal for new physics.

If we assume the excess is due to neutralino annihilation, then the statistical significance after one year of data taking with a large acceptance instrument, like AMS-02 13 with an acceptance of more than $0.04 \mathrm{~m}^{2} \mathrm{sr}$, will look like the points in Fig. 11. Here we assumed a boost factor as given by the fit to the AMS and HEAT data (Fig. 7). The resulting $\chi^{2}$ distribution is shown in Fig. 12 . Clearly, if the background estimates will be confirmed by accurate measurements of the electron spectrum, as will be done by the future experements, then the positron spectra can give a clear indication of neutralino annihilation with a rather precise determination of the neutralino mass.

We like to thank Drs. L. Bergström, J. Edsjö and P. Ullio for helpful discussions.

\section{REFERENCES}

1. S. W. Barwick et al. [HEAT Collaboration], Astrophys. J. 482 (1997) L191 arXiv:astro$\mathrm{ph} / 9703192$.

M. A. DuVernois et al., Astrophys. J. 559 (2001) 296.

2. J. Alcaraz et al. [AMS Collaboration], Phys. Lett. B 484 (2000) 10 [Erratum-ibid. B 495 (2000) 440].

3. E. A. Baltz et al., Phys. Rev. D 65 (2002) 063511 arXiv:astro-ph/0109318.

4. G. L. Kane, L. T. Wang and T. T. Wang, Phys. Lett. B 536 (2002) 263 arXiv:hep$\mathrm{ph} / 0202156$.

J. R. Ellis, J. L. Feng, A. Ferstl, K. T. Matchev and K. A. Olive, arXiv:astro$\mathrm{ph} / 0110225$.

M. Kamionkowski and M. S. Turner, Phys.
Rev. D 43 (1991) 1774.

5. W. de Boer, Prog. Part. Nucl. Phys. 33 (1994) 201 arXiv:hep-ph/9402266 and references therein.

6. W. de Boer et al., Eur. Phys. J. C 20 (2001) 689 arXiv:hep-ph/0102163.

7. W. de Boer et al., Z. Phys. C 67 (1995) 647 arXiv:hep-ph/9405342.

W. de Boer et al., Z. Phys. C 71 (1996) 415 arXiv:hep-ph/9603350.

W. de Boer et al., Phys. Lett. B 438 (1998) 281 arXiv:hep-ph/9805378.

8. CalcHEP, http://wwwzeuthen.desy.de/ pukhov/calchep.html,

A. Pukhov et al., arXiv:hep-ph/9908288.

9. DarkSUSY, P. Gondolo, et al., arXiv:astro$\mathrm{ph} / 0012234$.

10. A. W. Strong and I. V. Moskalenko, Adv. Space Res. 27 (2001) 717, arXiv:astro$\mathrm{ph} / 0101068]$.

11. A. H. Jaffe et al. [Boomerang Collaboration], Phys. Rev. Lett. 86 (2001) 3475 arXiv:astro$\mathrm{ph} / 0007333$.

12. V. Bonvicini et al. [PAMELA Collaboration], Nucl. Instrum. Meth. A 461 (2001) 262.

13. J. Alcaraz et al. [AMS Collaboration], Nucl. Instrum. Meth. A 478 (2002) 119. 